\title{
Invariant matrices and S-functions
}

\author{
By M. Zia-Ud-Din, University College, Swansea.
}

(Received 27th April, 1936. Read 1st May, 1936.)

1. It is known that an induced matrix of an induced matrix is expressible as the direct sum of invariant matrices, or more generally that an invariant matrix of an invariant matrix can be expressed as a direct sum $^{1}$ of invariant matrices. The spurs of the irreducible invariant matrices of a given matrix $A=\left[a_{s t}\right]$, are the $S$-functions ${ }^{2}$ of the latent roots of $A$.

If $A^{[\lambda]}=A^{\left[\lambda_{1}, \lambda_{1}, \ldots, \lambda_{p]}\right.}$ denotes the invariant matrix with spur $\left\{\lambda_{1}, \lambda_{2}, \ldots, \lambda_{p}\right\}$, then

$$
\left[A^{[\lambda]}\right]^{[\mu]}=\Sigma K_{\lambda \mu \nu} A^{[\nu]},
$$

where $\boldsymbol{\Sigma}$ stands for the direct sum.

2. From (1) D. E. Littlewood defines a new type of multiplication $^{3}$ for $S$-functions, namely

$$
\{\lambda\} \otimes\{\mu\}=\Sigma K_{\lambda \mu \nu}\{\nu\} .
$$

He has also shown that a problem involving the concomitants of polynomials may be solved by expressing the induced matrix of an induced matrix as the direct sum of invariant matrices. Hence the multiplication in (2) has especial importance when $\{\lambda\}$ and $\{\mu\}$ are the aleph symmetric functions, $h_{r}$ 's, and we therefore proceed to obtain a multiplication table for all cases when the weight of $\{\nu\}$ is $\leqq 12$, with the help of the tables of characters of the symmetric group.

2.1. Let $a, b, c, \ldots$ be the latent roots of $A$, and $h_{r}$ and $s_{r}$ be their aleph and power-sum symmetric functions. The spur of $A$ is clearly $h_{1}$.

If the matrix is in canonical form, the diagonal elements will be $a, b, c, \ldots$, the spur of the $m$ th induced matrix is $h_{m}=\Sigma a^{r} b^{b} c^{t} \ldots$

'Schur, "C'eber eine Klasse von Matrizen," Diss. Berlin (1901).

2 See D. E. Littlewood and Richardson, Phil. Trans. Roy. Soc. (A) 233 (1934), 107-115 for definition, and also Schur, loc. cit.

${ }^{3}$ D. E. Littlewood, Journal London Math. Soc. 11 (1936), 49.54. I am indebted to Mr Littlewood for suggesting the problem. 
Hence the canonical form contains elements $a^{r} b^{s} c^{\ell} \ldots$ in the leading diagonal1.

If $H_{r}$ and $Z_{r}$ are the aleph and the power-sum symmetric functions of the latent roots of this matrix, then $H_{r}$ is the spur of the $r$ th induced matrix, and hence we may write

$$
h_{m} \otimes h_{r}=H_{r}
$$

3. The problem now reduces to the expression of $H_{r}$ as the sum of $S$-functions of $a, b, c \ldots$

Now ${ }^{2}$

$$
Z_{1}=H_{1}=h_{m}=\frac{1}{m !} \Sigma h_{\rho} s_{\rho}
$$

where $h_{\rho}$ denotes the order of the class $\rho$ and $s_{\rho}=s_{1}^{p} s_{2}^{q} \ldots$, the class $\rho$ having $p$ cycles on 1 symbol, $q$ cycles on 2 and so on, and

$$
Z_{2}=\Sigma a^{2 r} b^{2 s} \ldots \ldots=\Sigma\left(a^{2}\right)^{r}\left(b^{2}\right)^{8} \ldots \ldots \text {; }
$$

hence $Z_{2}$ can be obtained from $Z_{1}$ by replacing $s_{m}$ by $s_{2 m}$. Similarly $Z_{r}$ can be obtained from $Z_{1}$ by replacing $s_{m}$ by $s_{r m}$.

$H_{r}$ is expressible as a function of $Z_{r}^{\prime}$, , which are known as functions of $s_{r}$ 's, and so $H_{r}$ is known in terms of $s_{r}$ 's.

To express $H_{r}$ as a linear function of $S$-functions, we read down the columns in the tables of characters to obtain the coefficients. Thus the multiplication $\{m\} \otimes\{r\}$ is obtained and the table constructed.

As a check for the calculations we use

$$
\frac{(m r) !}{(m !)^{r} r !}=\Sigma K_{m r} \chi_{0}^{(v)}
$$

The check may be derived as follows. Putting $s_{1}=1, s_{2}=s_{3}=\ldots=0$, we have

$$
\begin{aligned}
& \text { Also from }{ }^{3} \\
& \text { we get } \\
& \begin{array}{l}
Z_{1}=\frac{1}{m !}, \quad Z_{2}=Z_{3}=\ldots=0 \\
H_{r}=\frac{1}{r !} Z_{1}^{r}=\frac{1}{r !(m !)^{r}} .
\end{array} \\
& n !\{\lambda\}=\Sigma h_{\rho} \chi_{\rho}^{(\lambda)} S_{\rho}, \\
& \{\lambda\}=\frac{1}{n !} \chi_{0}^{(\lambda)} .
\end{aligned}
$$

1 Schur, op. cit., pp. 10, 11.

2 D. E. Littlewood and A. R. Richardson, op. cit., p. 109.

${ }^{3}$ Littlewood and Richardson, Phil. Trans. Roy. Soc. (A) 233 (1934), 109. 
Since $H_{r}$ is expressible as a sum of $S$-functions, therefore

$$
H_{r}=\frac{1}{(m r) !} \Sigma K_{r m v} \chi_{0}^{(v)}
$$

which combined with (3) gives the check.

4. The actual working will be shown by obtaining $\{2\} \otimes\{3\}$.

$$
\begin{gathered}
Z_{1}=H_{1}=h_{2}=\frac{1}{2}\left(s_{1}^{2}+s_{2}\right) \\
Z_{2} \quad=\frac{1}{2}\left(s_{2}^{2}+s_{4}\right) \\
Z_{3} \quad=\frac{1}{2}\left(s_{3}^{2}+s_{6}\right) \\
H_{3}=\frac{1}{6}\left(Z_{1}^{3}+3 Z_{1} Z_{2}+2 Z_{3}\right) . \\
48 H_{3}=s_{1}^{6}+3 s_{1}^{4} s_{2}+6 s_{1}^{2} s_{4}+9 s_{1}^{2} s_{2}^{2} \\
\quad+8 s_{6}+6 s_{2} s_{4}+7 s_{2}^{3}+8 s_{3}^{2} .
\end{gathered}
$$

From the table of degree $6, H_{3}$ turns out to be

$$
\{6\}+\{42\}+\left\{2^{3}\right\} \text {. }
$$

Therefore

$$
\{2\} \otimes\{3\}=\{6\}+\{42\}+\left\{2^{3}\right\} .
$$

5. With the help of the tables ${ }^{1}$ of the group characters the following multiplication table is constructed.

$$
\begin{aligned}
& \{2\} \otimes\{2\}=\{4\}+\left\{2^{2}\right\} . \\
& \{2\} \otimes\{3\}=\{6\}+\{42\}+\left\{2^{3}\right\} . \\
& \{2\} \otimes\{4\}=\{8\}+\{62\}+\left\{4^{2}\right\}+\left\{42^{2}\right\}+\left\{2^{4}\right\} . \\
& \{2\} \otimes\{5\}=\{10\}+\{82\}+\{64\}+\left\{62^{2}\right\}+\left\{2^{5}\right\}+\left\{4^{2} 2\right\}+\left\{42^{3}\right\} . \\
& \{2\} \otimes\{6\}=\{12\}+\{10,2\}+\{84\}+\left\{82^{2}\right\}+\left\{6^{2}\right\} \\
& \quad+\{642\}+\left\{62^{3}\right\}+\left\{4^{3}\right\}+\left\{4^{2} 2^{2}\right\}+\left\{42^{4}\right\}+\left\{2^{6}\right\} . \\
& \{3\} \otimes\{2\}=\{6\}+\{42\} . \\
& \{3\} \otimes\{3\}=\{9\}+\{72\}+\{63\}+\left\{52^{2}\right\}+\left\{4^{2} 1\right\} . \\
& \{3\} \otimes\{4\}=\{12\}+\{10,2\}+\{93\}+\{84\}+\left\{82^{2}\right\}+\{741\}+\{732\} \\
& \quad+\left\{6^{2}\right\}+\{642\}+\left\{62^{3}\right\}+\{5421\}+\left\{4^{3}\right\} . \\
& \{4\} \otimes\{2\}=\{8\}+\{62\}+\left\{4^{2}\right\} . \\
& \{4\} \otimes\{3\}=\{12\}+\{10,2\}+\{93\}+\{84\}+\left\{82^{2}\right\}+\{741\} \\
& \{5\} \otimes\{2\}=\{10\}+\{82\}+\{64\} . \\
& \{6\} \otimes\{2\}=\{12\}+\{10,2\}+\{84\}+\left\{6^{2}\right\} .
\end{aligned}
$$

1 Tables as far as 10th degree will be found in D. E. Littlewood's paper, Proc. London Math. Soc. (2) 39 (1935), 177-183. The 12th degree table is to appear in Proc. London Math. Soc. 